\title{
A comparison between the mechanical and thermal properties of single-walled carbon nanotubes and boron nitride nanotubes
}

Ting Li, Zhenan Tang, Zhengxing Huang*, Jun Yu

School of Electronic Science and Technology, Key Lab. of Liaoning for Integrated Circuits Technology, Dalian University of Technology, Dalian 116024, China.

Keywords:

Carbon nanotubes; boron nitride nanotubes; mechanical properties; thermal properties; molecular dynamics.

\section{ABSTRACT}

Carbon nanotubes (CNTs) are semimetallic while boron nitride nanotubes (BNNTs) are wide band gap insulators. Despite the discrepancy in their electrical properties, a comparison between the mechanical and thermal properties of CNTs and BNNTs has a significant research value for their potential applications. In this work, molecular dynamics simulations are performed to systematically investigate the mechanical and thermal properties of CNTs and BNNTs. The calculated Young's modulus is about 1.1 TPa for CNTs and 0.72 TPa for BNNTs under axial compressions. The critical bucking strain and maximum stress are inversely proportional to both diameter and length-diameter ratio and CNTs are identified axially stiffer than BNNTs. Thermal conductivities of $(10,0)$ CNTs and $(10,0)$ BNNTs follow similar trends with respect to length and temperature and are lower than that of their two-dimensional counterparts, graphene nanoribbons (GNRs) and BN nanoribbons (BNNRs), respectively. As the temperature falls below $200 \mathrm{~K}(130 \mathrm{~K})$ the thermal conductivity of BNNTs (BNNRs) is larger than that of CNTs (GNRs), while at higher temperature it is lower than the latter. In addition, thermal conductivities of a $(10,0) \mathrm{CNT}$ and a $(10,0)$ BNNT are further studied and analyzed under various axial compressive strains. Low-frequency phonons which mainly come from flexure modes are believed ${ }^{*}$ Corresponding author.

E-mail address: huangzx@dlut.edu.cn (Z. X. Huang). 
to make dominant contribution to the thermal conductivity of CNTs and BNNTs.

\section{Introduction}

Carbon nanotubes (CNTs), one-dimensional (1D) counterpart of graphene, have aroused much research interest due to their particular and distinguished material properties [1-3]. Boron nitride nanotubes (BNNTs) have attracted attention in recent years because they have a structual analogy to CNTs and possese many unique properties and potential applications in nanotechnologies comparable with CNTs [4-6]. In regard to electronic structure, CNTs are metallic or semiconducting depending on chiralities, whereas BNNTs are normally electrically insulating with a wide band gap of $5.0-6.0 \mathrm{eV}$, independent of chiralities. Both CNTs and BNNTs have superb mechanical properties and high thermal conductivities. Besides that BNNTs possess better thermal and oxidation stability than CNTs [7-10]. CNTs and BNNTs are promising components as reinforcements in composites for enhenced mechanical and thermal properties in micro/nano-electro-mechanical devices [11-18]. Compared to CNTs, BNNTs have remained much less explored, which is primarily due to the lack of successful experiments performed on BNNTs. Till now, the growth of highly pure single-walled BNNTs at large quantities remains a challenge and the well-established synthetic routes of CNTs and graphene formation do not effeciently work for the BN nanostructures [19-23].

For mechanical properties, The commonly accepted value of the Young's modulus for CNTs is about $0.9 \mathrm{TPa}[24,25]$, and it is generally true that the Young's modulus for a BNNT is smaller than for a CNT of same radius [26]. Yan et al. [27] predicted the elastic properties of singled-walled BNNTs using an atomistic-continuum approach and found that chirality has a negligible effect on the axial Young's modulus of BNNTs (0.91-0.975 TPa) but plays a remarkable role in the circumferential and shear modulus. On the basis of the molecular mechanics approach, the Young's modulus of BNNTs subjected to axial loadings are 0.17-0.27 TPa [28]. However, experimentally, both CNTs and BNNTs' Young's modulus vary in samples 
fabricated under different conditions, and the specific discrepencies in the mechanical properties of CNTs and BNNTs are still not quite clear.

In regard of thermal properties, discrepancies in thermal conductity magnitude with experimental data are primarily attributed to the limited size effects in the simulations and the thermal conductivity of CNTs increases with nanotube length, varying from about $10 \mathrm{~W} / \mathrm{m} / \mathrm{K}$ to $375 \mathrm{~W} / \mathrm{m} / \mathrm{K}$ [29]. By using the kinetic theory, Jiang et al. [30] found that the two flexure modes of the single-walled BNNT make dominant contribution to the thermal conductivity. Sevik et al. [31] performed equilibrium molecular dynamics (MD) simulations to investigate the thermal transport properties of $\mathrm{BN}$ nanostructures and the results show that $\mathrm{BN}$ nanostructures have considerably high thermal conductivities but are still quite lower than carbon-based counterparts. Despite the efforts in the studies of the thermal properties of CNTs and BNNTs, a systematic comparion between them is lacking, expecially when the system is under axial compressions.

In this work, MD simulations are performed using the package LAMMPS to systematically investigate the machanical and thermal properties of CNTs and BNNTs under the same calculation conditions. The Young's modulus, critical bucking strain and maximum stress under axial compressions are studied for CNTs and BNNTs with various diameters and length-diameter ratios. For a better understanding of the characteristics of the thermal properties of $\mathrm{C}$ and $\mathrm{BN}$ nanotubes, thermal conductivities of $(10,0) \mathrm{CNTs}$ and $(10,0)$ BNNTs are calculated as a function of length and temperature, in comparison with that of their two-dimensional (2D) counterparts, graphene nanoribbons (GNRs) and BN nanoribbons (BNNRs), respectively. Moreover, thermal conductivities of a $(10,0) \mathrm{CNT}$ and a $(10,0) \mathrm{BNNT}$ are further studied and analyzed when loaded by various axial compressive strains. Results in this work provide fundamental knowledge for the applications of CNTs and BNNTs in micro/nano-electro-mechanical devices.

\section{Mechanical properties}


$(10,0),(10,10)$ and $(20,0) \mathrm{C}$ and $\mathrm{BN}$ nanotubes are used in the simulations, and the length of C-C bonding and B-N bonding are $1.42 \AA$ and $1.45 \AA$, respectively. The parametrized Tersoff potentials obtained by Lindsay et al. [32] and Sevik et al. [31] are employed to describe the interactions between $\mathrm{C}-\mathrm{C}$ atoms and $\mathrm{B}-\mathrm{N}$ atoms, respectively, which yield very accurate results for mechanical and thermodynamic properties of $\mathrm{C}$ and $\mathrm{BN}$ low-dimensional nanostructures [33-36]. During the simulation, an integration time step of $0.4 \mathrm{fs}$ is used and periodic boundary conditions (PBC) are applied in the three directions with vacuum slabs large enough in the radial directions of the nanotube to eliminate the effects of neighboring atoms. Applying PBC along the loading direction could decrease the finite length effects and provide accurate uniaxial stress conditions [37]. In the beginning, optimization procedures are carried out for the simulation model to relax artificial constrains applied on the model during construction. Then the system is set at $300 \mathrm{~K}$ within the NVT ensemble for 200 ps. After the equilibration, the axial compressive strains are applied by compressing one end of the nanotube with a constant engineering strain value of $0.1 \%$ each time, followed by an equilibration process for $40 \mathrm{ps}$. The compression-equilibration process totally repeats 100 times and the final axial compressive strain of the system is $10 \%$. During the loading process, stress of the system is recorded as a function of strain every $40 \mathrm{ps}$ in order to obtain the stress-strain curves. The Young's modulus is evaluated by calculation of the slope of the linear part of stress-strain relation. 

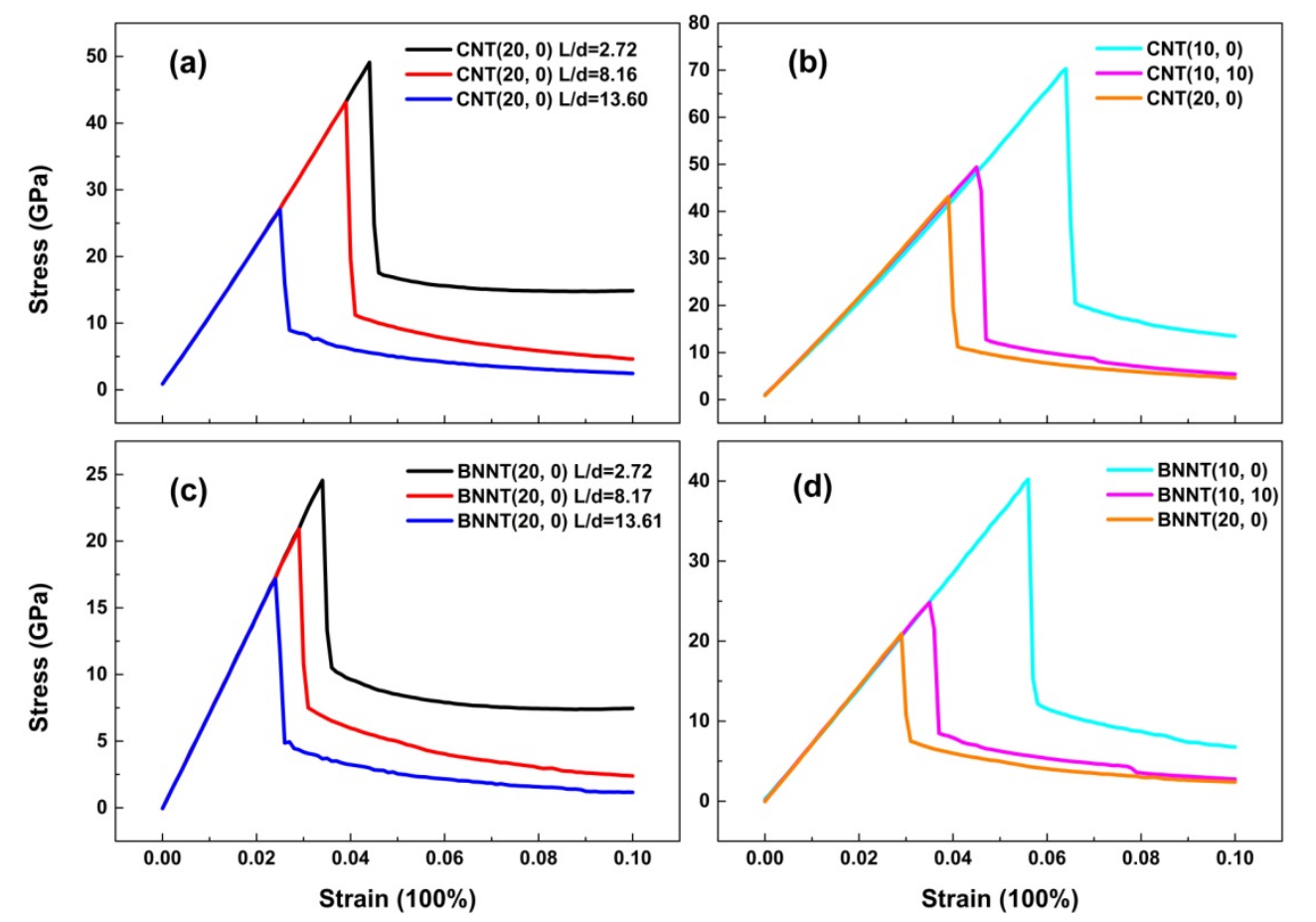

Fig. 1. Stress-strain curves of (a) $(20,0)$ CNTs with various length-diameter ratios, (b) $(10,0),(10$, $10)$ and $(20,0)$ CNTs with a length-diameter ratio of 8.16 , (c) $(20,0)$ BNNTs with various length-diameter ratios and (d) $(10,0),(10,10)$ and $(20,0)$ BNNTs with a length-diameter ratio of 8.17 .

The stress-strain curves of CNTs and BNNTs with various diameters (d) and length-diameter ratios (L/d) are shown in Fig. 1. At the first stage, stress linearly increases with increased strains. When the value of strain is increased to the critical bucking strain, stress drops sharply and then decreases very slowly with increasing strains. Finally, stress remains almost unchanged with further compressions and proceeds to attain a constant value. The stress-strain curves of CNTs and BNNTs are similar to the stress-strain response of pristine graphene [33,34], phagraphene [33], amorphous graphene [34] and nitrogenated holey graphene [38] under tensile strains obtained by MD simulations in previous studies. The Young's modulus (Y), critical bucking strain $(\varepsilon)$ and maximum stress $(\sigma)$ of various CNTs and BNNTs are listed in Table1 and Table 2, respectively. The calculated Young's modulus is about 1.1 TPa for CNTs and $0.72 \mathrm{TPa}$ for BNNTs, independent of chiralities, diameters and length-diameter ratios, and the Young's modulus of BNNTs is about $34.5 \%$ smaller 
than that of CNTs. The critical bucking strain and maximum stress for a CNT is larger than for a BNNT with same diameter and length-diameter ratio, confirming the fact that CNTs are axially stiffer than BNNTs. For both CNTs and BNNTs, the critical bucking strain and maximum stress are inversely proportional to diameter and length-diameter ratio, indicating size dependence of the axial deformation behaviors of $\mathrm{C}$ and $\mathrm{BN}$ nanotubes.

Table 1. Young's modulus, critical bucking strain and maximum stress for various CNTs.

\begin{tabular}{c|c|c|c|c|c}
\hline $\mathrm{CNT}$ & $\mathrm{d}(\AA)$ & $\mathrm{L} / \mathrm{d}$ & $\mathrm{Y}(\mathrm{TPa})$ & $\varepsilon$ & $\sigma(\mathrm{GPa})$ \\
\hline$(10,0)$ & 7.83 & 8.16 & 1.08 & 0.064 & 70.3 \\
\hline$(10,10)$ & 13.56 & 8.16 & 1.07 & 0.045 & 49.4 \\
\hline$(20,0)$ & 15.66 & 2.72 & 1.07 & 0.044 & 49.1 \\
\hline$(20,0)$ & 15.66 & 8.16 & 1.07 & 0.039 & 43.1 \\
\hline$(20,0)$ & 15.66 & 13.60 & 1.07 & 0.025 & 27.0 \\
\hline
\end{tabular}

Table 2. Young's modulus, critical bucking strain and maximum stress for various BNNTs.

\begin{tabular}{c|c|c|c|c|c}
\hline BNNT & $\mathrm{d}(\AA)$ & $\mathrm{L} / \mathrm{d}$ & $\mathrm{Y}(\mathrm{TPa})$ & $\varepsilon$ & $\sigma(\mathrm{GPa})$ \\
\hline$(10,0)$ & 7.99 & 8.17 & 0.714 & 0.056 & 40.2 \\
\hline$(10,10)$ & 13.83 & 8.17 & 0.715 & 0.035 & 24.8 \\
\hline$(20,0)$ & 15.98 & 2.72 & 0.721 & 0.034 & 24.6 \\
\hline$(20,0)$ & 15.98 & 8.17 & 0.723 & 0.029 & 20.9 \\
\hline$(20,0)$ & 15.98 & 13.61 & 0.721 & 0.024 & 17.2 \\
\hline
\end{tabular}

Fig. 2 shows the snapshots of the axial deformation behaviors of $(20,0)$ CNTs with different length-diameter ratios under various axial compressive strains. At first, the structural deformation of $\mathrm{CNT}$ is unobvious until the value of strain is increased to its critical bucking strain. The CNT with a large length-diameter ratio begins to buckle first due to its small value of critical buckling strain. For example, the CNT in Fig. 2(c) has shown deformation behaviors when the strain is 0.03 , whereas the deformation of CNTs in Fig. 2(a) and (b) are not obvious. When the strain is 0.04, the structural deformation of the CNT in Fig.2 (b) can be observed. And the deformation 
behaviors become obvious for all the CNTs when the strain reached to 0.05 . The deformation behaviors of BNNTs are similar to that of CNTs since they have a structual analogy to CNTs. However, the BNNT begins to show buckling defomations earlier than CNT because the critical bucking strain for a BNNT is smaller than for a CNT with same diameter and length-diameter ratio.

(a)

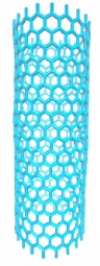

(b)

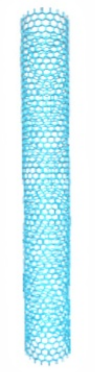

(c)
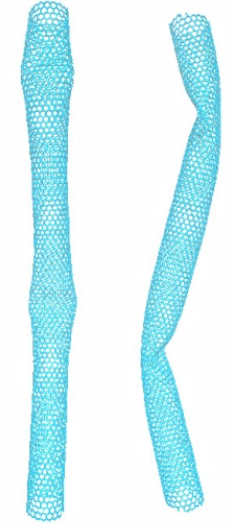

0.03
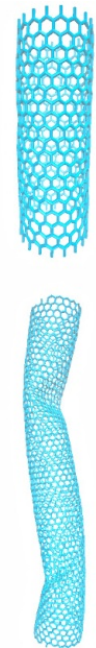
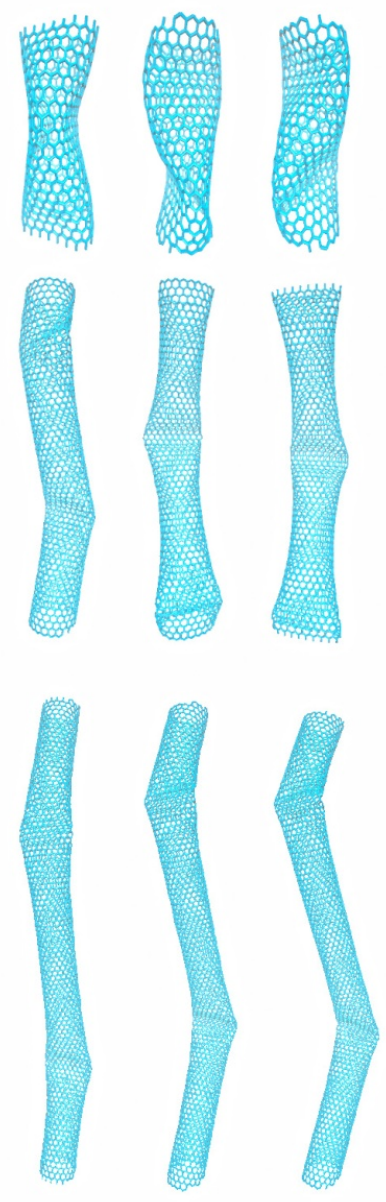

0.04

0.05

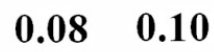

Fig. 2. Snapshots of the deformation behaviors of $(20,0)$ CNTs with a length-diameter ratio of (a) 2.72, (b) 8.16 and (c) 13.60 loaded by various axial compressive strains.

\section{Thermal properties}

Non-equilibrium MD (NEMD) method is performed to calculate the thermal conductivity of $(10,0)$ CNTs and $(10,0)$ BNNTs as well as their $2 \mathrm{D}$ counterparts, armchair GNRs and armchair BNNRs, respectively. The potential parameters, 
boundary conditions and time step are the same with calculations of mechanical properties. The simulation box along the longitudinal direction is divided into $\mathrm{N}(\mathrm{N} \geq$ 50) slabs. First, the system is relaxed at a temperature T within the NVT ensemble for 320 ps. Then the system is turned into NVE ensemble for 80 ps. After the equilibration, a temperature difference is applied between the hot reservoir (placed at one quarter of the length) and the cold reservoir (placed at three quarters of the length). In this study, the temperatures at the hot reservoir and the cold reservoir are set to $(\mathrm{T}+\Delta \mathrm{T}) \mathrm{K}$ and $(\mathrm{T}-\Delta \mathrm{T}) \mathrm{K}$, respectively, using the Langevin [39] thermostat. $\Delta \mathrm{T}$ is set as 0.4 times of $\mathrm{T}$ in the simulations to ensure the accuracy in fitting the temperature gradient. Then the non-equilibrium steady state heat transfer can be achieved after $400 \mathrm{ps}$ of the exchanging process within the NVE ensemble, when a temperature gradient is established along the longitudinal direction. The energy of the simulation box remains constant and a constant heat flux is applied throughout the sample. The heat flux is a constant quantity of kinetic energy transferring from the atoms in the hot reservoir to those in the cold reservoir at each simulation time step. The thermal conductivity, $k$, of the system could be calculated using a 1D form of the Fourier law [40]:

$k=-\frac{J}{A \times \nabla T}$

where $J$ is the applied heat flux, $\nabla T$ is the established temperature gradient and $A$ is the cross sectional area of heat transfer. In this work, $0.34 \mathrm{~nm}$ is used as the thickness of $1 \mathrm{D}$ and $2 \mathrm{D} \mathrm{C}$ and $\mathrm{BN}$ nanostructures. The temperature at each slab is computed as follows [40]:

$T_{i}=\frac{2}{3 N k_{B}} \sum_{j} \frac{p_{j}^{2}}{2 m_{j}}$

where $T_{i}$ is the temperature of the ith slab, $N$ is the number of atoms in this slab, $k_{B}$ is the Boltzman's constant, $m_{j}$ and $p_{j}$ are the atomic mass and momentum of atom $j$, respectively. During the last 400 ps, the averaged temperatures at each slab are computed. This way, a linear temperature gradient is established in the middle of the sample. By calculating the established $\nabla T$, thermal conductivity is obtained using 
Eq. (1).

The effects of length and temperature on lattice thermal conductivity are investigated. Fig. 3 indicates that length has a strong influence on the thermal conductivity of low-dimensional $\mathrm{C}$ and $\mathrm{BN}$ nanostructures. For nanomaterials, thermal energies transport in a ballistic way instead of diffusive transport when its feature size are comparable to phonon mean free path (MFP), which, for example, is of the order of $775 \mathrm{~nm}$ in graphene [41], much larger than the size of our simulation cells. On the basis of the kinetic theory of phonon transport [42], the thermal conductivity is proportional to the specific heat $c_{v}$, group velocity $v_{g}$, and MFP $\Lambda$ of thermal energy carriers:

$k \propto c_{v} v_{g} \Lambda$

and the effective MFP $\Lambda$ is given by

$$
\frac{1}{\Lambda}=\frac{1}{\Lambda_{p-p}}+\frac{1}{\Lambda_{L}}
$$

where $\Lambda_{p-p}$ is phonon-phonon scattering length and $\Lambda_{L}$ is scattering length due to the boundaries in a finite system, which can be approximated to the length of the simulation box. Therefore, the thermal conductivity satisfies the relation:

$$
\frac{1}{k} \propto \frac{1}{\Lambda_{L}}+\frac{1}{\Lambda_{p-p}}
$$

Our calculation results confirm this scaling relation and the thermal conductivity of CNTs, BNNTs, GNRs and BNNRs with an infinite length at $300 \mathrm{~K}$ are estimated to be $901 \mathrm{~W} / \mathrm{m} / \mathrm{K}, 369 \mathrm{~W} / \mathrm{m} / \mathrm{K}, 1316 \mathrm{~W} / \mathrm{m} / \mathrm{K}$ and $526 \mathrm{~W} / \mathrm{m} / \mathrm{K}$, respectively, using the scaling approach. The values of thermal conductivity with an infinite length are still small compared to those from experiments, which are due to the small radius of nanotubes and width of nanoribbons used in the simulations. 


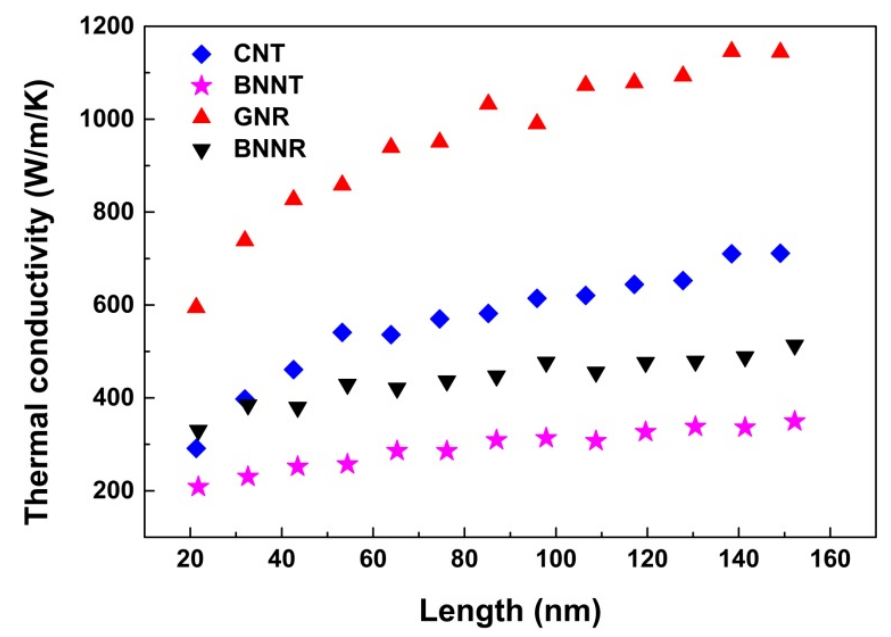

Fig. 3. Length dependence of room temperature thermal conductivity of $(10,0)$ CNTs and $(10,0)$ BNNTs, compared with equivalent armchair GNRs and armchair BNNRs.

In Fig. 4, thermal conductivity decays exponentially with increased temperatures in the range of $100 \mathrm{~K}$ to $1200 \mathrm{~K}$. Values of thermal conductivity below $100 \mathrm{~K}$ are not calculated in this work for the sake of calculation accuracy using the NEMD method. In fact, at low temperatures, thermal conductivity of nanomaterials increases with increasing cryogenic temperatures, which are dominated by the material's specific heat. Then, in a range of transition temperatures, the increase in specific heat begins to moderate while phonon-phonon scattering becomes prevalent. The peak thermal conductivity occurs in this region. At high temperatures, thermal conductivity becomes to decrease as specific heat becomes constant while the phonon-phonon scattering rate continues to increase because more high-frequency phonons are populated and thus available to be scattered. Thermal transport regimes in nanomaterials are just as Fig. 3 of [43] indicated. 


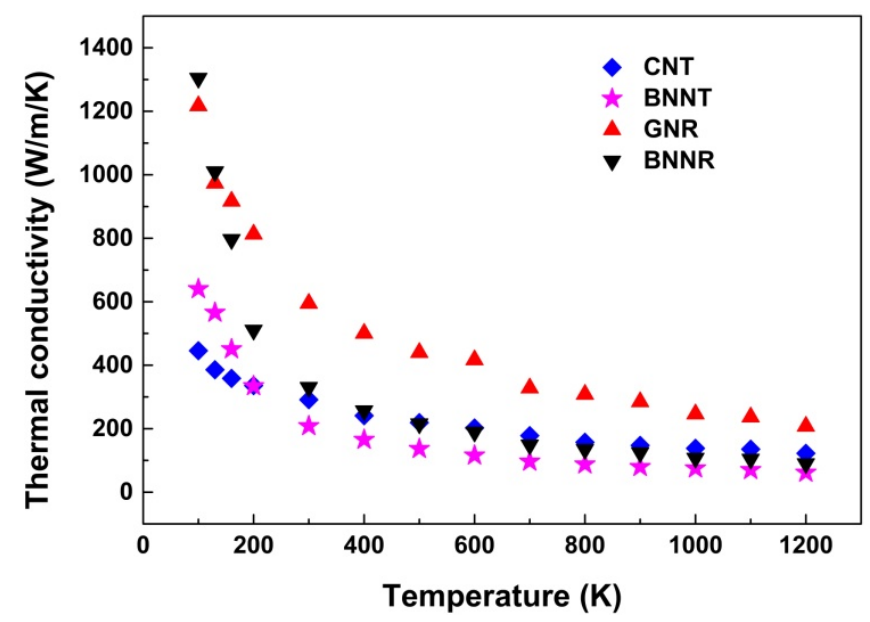

Fig. 4. Temperature dependence of lattice thermal conductivity of a $(10,0) \mathrm{CNT}$ and a $(10,0)$ BNNT, compared with their 2D counterparts, an armchair GNR and an armchair BNNR. All the systems are comprised of 2000 atoms with a longitudinal length of $\sim 21 \mathrm{~nm}$.

The thermal conductivity of CNTs and BNNTs at different temperatures are lower than their 2D counterparts, GNRs and BNNRs, respectively, and the difference in thermal conductivity between CNTs and GNRs is larger than that between BNNTs and BNNRs. As the temperature falls below $200 \mathrm{~K}(130 \mathrm{~K})$ the thermal conductivity of BNNTs (BNNRs) is larger than that of CNTs (GNRs), while at higher temperature it is lower than the latter, which are possibly attributed to the following reasons: First, thermal conductivity is dominated by specific heat at low temperatures and $\mathrm{BN}$ nanostructures are reported to have a larger specific heat compared with their carbon analogs [44]. Secondly, the different atomic mass fluctuations and force constants in $\mathrm{BN}$ nanostructures leads to stronger phonon-phonon scattering than carbon nanostructures at high temperatures. Therefore, the thermal conductivity of BN nanostructures is larger than that of their carbon analogs at low temperatures, while at higher temperature it is lower than the latter.

\section{Thermal conductivity under axial compressions}

Being loaded by axial compressions are inevitable when CNTs/BNNTs are used as components in the composites for enhenced thermal properties of the device, which 
can also strongly influence a nanotube's thermal conductivity. Shown in Fig. 5 is the axial compressive strain dependence of the thermal conductivity of a $(10,0)$ CNT and a $(10,0)$ BNNT. As one can see, unlike BNNT, the thermal conductivity of which decreases with increased compressive strains, CNT gets it maximum value of thermal conductivity when the strain is 0.01 and then the thermal conductivity of which decreases with further increased strains on the whole. Variations in compressive strains affect the lattice anharmonicity and stronger phonon-phonon scattering occurs in general with increased strains, leading to a decrease in thermal conductivity on the whole. To further explain the underlying mechanisms of the influences of compressive strains on the thermal conductivity of CNT and BNNT, the density of states (DOS) that describes the distribution of vibration power at different frequencies is calculated, which is obtained from the Fourier transform of the velocity autocorrelation function $[29,45]$. It is observed from phonon DOS in Fig. 6 that the peak of high-frequency phonons for both CNT and BNNT appear at $48 \mathrm{THz}$, while the peak of low-frequency phonons for CNT and BNNT are about $12 \mathrm{THz}$ and $9 \mathrm{THz}$, respectively. The discrepancy in the distributions of low-frequency phonons may be the cause of the difference between the thermal conductivity of CNT and BNNT. Both the peaks of low-frequency and high-frequency phonons are suppressed by axial compressions on the whole, leading to a decrease in the thermal conductivity of CNT and BNNT with increased compressive strains. Specifically, unlike the BNNT, the thermal conductivity of CNT is maximum when the strain is 0.01 , corresponding to a largest peak of the low-frequency phonons in DOS, while the peak of high-frequency phonons is suppressed compared to that without compressions. Therefore, low-frequency phonons mainly coming from flexure modes are indicated to make dominant contribution to the thermal conductivity of CNT and BNNT. 


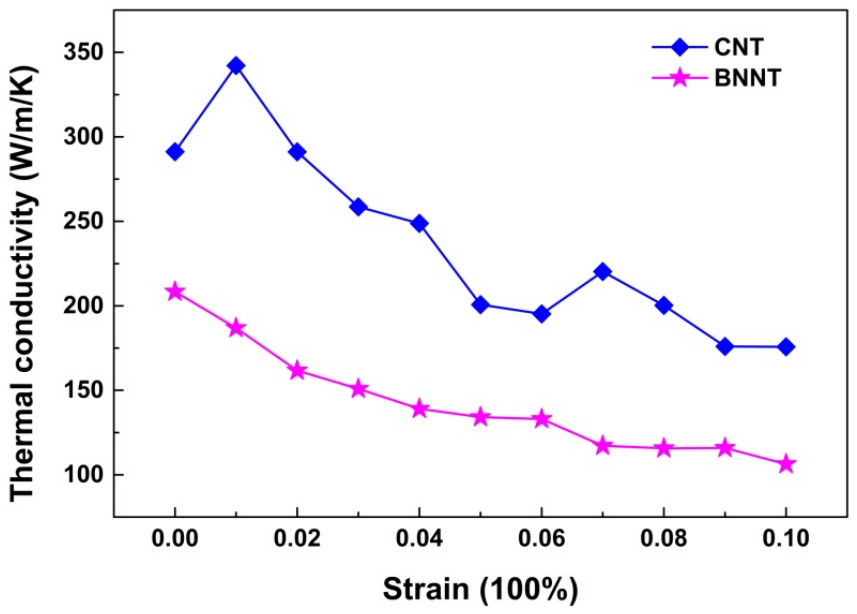

Fig. 5. Axial compressive strain dependence of the thermal conductivity of a $(10,0) \mathrm{CNT}$ and a $(10,0)$ BNNT at room temperature, with a length of $\sim 21 \mathrm{~nm}$.

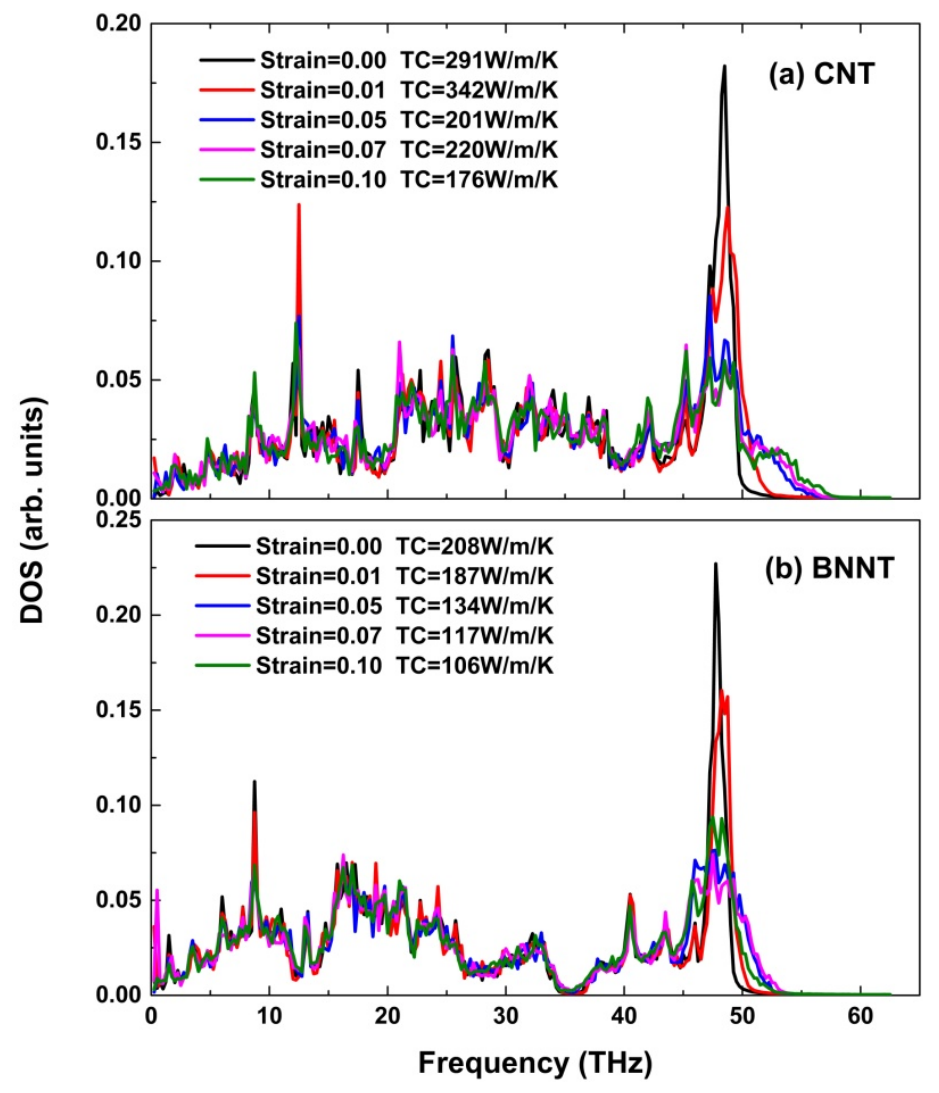

Fig. 6. DOS of (a) a $(10,0) \mathrm{CNT}$ and (b) a $(10,0)$ BNNT with variations in axial compressive strains at room temperature.

\section{Conclusions}


In summary, a comparison between the mechanical and thermal properties of CNTs and BNNTs are systematically studied by MD simulations using the LAMMPS package. For mechanical properties, stress-strain curves and deformation behaviors of CNTs and BNNTs under axial compressive strains are explored, with various diameters and length-diameter ratios considered. The calculated Young's modulus is about 1.1 TPa for CNTs and 0.72 TPa for BNNTs. The critical bucking strain and maximum stress are inversely proportional to both diameter and length-diameter ratio of the nanotubes and CNTs are identified axial stiffer than BNNTs. In regard to thermal properties, the thermal conductivity of $(10,0)$ CNTs and $(10,0)$ BNNTs, as well as equivalent GNRs and BNNRs, are calculated using NEMD method. The characteristics of length and temperature dependence on thermal conductivity exhibit ballistic thermal transport in nanomaterials and the calculated results indicate the thermal conductivity of CNTs and BNNTs are lower than their 2D counterparts, GNRs and BNNRs, respectively. As the temperature falls below $200 \mathrm{~K}(130 \mathrm{~K})$ the thermal conductivity of BNNTs (BNNRs) is larger than that of CNTs (GNRs), while at higher temperature it is lower than the latter. It is because thermal conductivity is dominated by specific heat at low temperatures while phonon-phonon scattering is prevailing at high temperatures. Furthermore, the thermal conductivity of a $(10,0)$ CNT and a $(10,0)$ BNNT under various axial compressions are studied and it is demonstrated that low-frequency phonons which mainly come from flexure modes make dominant contribution to the thermal conductivity of CNT and BNNT. These results provide useful guidelines for the potential applications of CNTs, BNNTs and their composites in micro/nano-electro-mechanical devices.

\section{Acknowledgements}

This work was supported by the National Science Foundation of China under grant No. 61131004 and the Fundamental Research Funds for the Central Universities (DUT14LAB11). 


\section{References}

[1] S. Iijima, Helical microtubules of graphitic carbon, Nature 354 (1991) 56-58.

[2] D.S. Bethune, C.H. Kiang, M.S. De Vries, G. Gorman, R. Savoy, J. Vazquez, R. Beyers,

Cobalt-catalysed growth of carbon nanotubes with single-atomic-layer walls, Nature 363 (1993) 605-607.

[3] S. Iijima, T. Ichihashi, Single-shell carbon nanotubes of 1-nm diameter, Nature 363 (1993) 603-605.

[4] A. Rubio, J.L. Corkill, M.L. Cohen, Theory of graphitic boron nitride nanotubes, Phys. Rev. B 49 (1994) 5081.

[5] X. Blase, A. Rubio, S.G. Louie, M.L. Cohen, Stability and Band Gap Constancy of Boron Nitride Nanotubes, Europhysics Letters 28 (1994) 335-340.

[6] N.G. Chopra, R.J. Luyken, K. Cherrey, V.H. Crespi, M.L. Cohen, S.G. Louie, A. Zettl, Boron nitride nanotubes, Science 269 (1995) 966-967.

[7] C. Zhi, Y. Bando, C. Tang, D. Golberg, Boron nitride nanotubes, Materials Science and Engineering: R: Reports 70 (2010) 92-111.

[8] A.A. Balandin, Thermal properties of graphene and nanostructured carbon materials, Nature Materials 10 (2011) 569-581.

[9] J.H. Yuan, K.M. Liew, Effects of boron nitride impurities on the elastic properties of carbon nanotubes, Nanotechnology 19 (2008) 445703.

[10] L. Vaccarini, C. Goze, L. Henrard, E. Hernandez, P. Bernier, A. Rubio, Mechanical and electronic properties of carbon and boron-nitride nanotubes, Carbon 38 (2000) 1681-1690.

[11] H. Huang, C.H. Liu, Y. Wu, S. Fan, Aligned carbon nanotube composite films for thermal management, Advanced Materials 17 (2005) 1652-1656.

[12] A.M. Marconnet, N. Yamamoto, M.A. Panzer, B.L. Wardle, K.E. Goodson, Thermal conduction in aligned carbon nanotube-polymer nanocomposites with high packing density, ACS Nano 5 (2011) $4818-4825$.

[13] A. Kumar, M.R. Maschmann, S.L. Hodson, J. Baur, T.S. Fisher, Carbon nanotube arrays decorated with multi-layer graphene-nanopetals enhance mechanical strength and durability, Carbon 84 (2015) 236-245. 
[14] J.N. Coleman, U. Khan, W.J. Blau, Y.K. Gun'ko, Small but strong: a review of the mechanical properties of carbon nanotube-polymer composites, Carbon 44 (2006) 1624-1652.

[15] D. Lahiri, V. Singh, A.P. Benaduce, S. Seal, L. Kos, A. Agarwal, Boron nitride nanotube reinforced hydroxyapatite composite: Mechanical and tribological performance and in-vitro biocompatibility to osteoblasts, Journal of the Mechanical Behavior of Biomedical Materials 4 (2011) 44-56.

[16] H.W. Yap, R.S. Lakes, R.W. Carpick, Mechanical instabilities of individual multiwalled carbon nanotubes under cyclic axial compression, Nano Letters 7, (2007) 1149-1154.

[17] R. Arenal, M.S. Wang, Z.Xu, A. Loiseau, D. Golberg, Young modulus, mechanical and electrical properties of isolated individual and bundled single-walled boron nitride nanotubes, Nanotechnology $22(2011) 265704$.

[18] Y. Huang, J. Lin, J. Zou, M.S. Wang, K. Faerstein, C.C. Tang, Y. Bando, D. Golberg, Thin boron nitride nanotubes with exceptionally high strength and toughness, Nanoscale 5 (2013) 4840-4846.

[19] D. Golberg, Y. Bando, Y. Huang, T. Terao, M. Mitome, C.C. Tang, C.Y. Zhi, Boron Nitride Nanotubes and Nanosheets, ACS Nano 4 (2010) 2979-2993.

[20] D. Golberg, Y. Bando, C.C. Tang, C.Y. Zhi, Boron nitride nanotubes, Advanced Materials 19 (2007) 2413-2432.

[21] A.T. Matveev, K.L. Firestein, A.E. Steinman, A.M. Kovalskii, O.I. Lebedev, D.V. Shtansky, D. Golberg, Boron nitride nanotube growth via boron oxide assisted chemical vapor transport-deposition process using $\mathrm{LiNO}_{3}$ as a promoter, Nano research 8 (2015) 2063-2072.

[22] A. Pakdel, C.Y. Zhi, Y. Bando, T. Nakayama, D. Golberg, Nanotechnology 23 (2012) 215601.

[23] L. Liu, J. Park, D.A. Siegel, K.F. McCarty, K.W. Clark, W. Deng, L. Basile, J.C. Idrobo, A.P. Li, G. Gu, A comprehensive analysis of the CVD growth of boron nitride nanotubes, Science 343 (2014) $163-167$.

[24] M. Meo, M. Rossi, Prediction of Young's modulus of single wall carbon nanotubes by molecular-mechanics based finite element modelling, Composites Science and Technology 66 (2006) 1597-1605.

[25] B.G. Demczyk, Y.M. Wang, J. Cumings, M. Hetman, W. Han, A. Zettl, R.O. Ritchie, Direct mechanical measurement of the tensile strength and elastic modulus of multiwalled carbon nanotubes, Materials Science and Engineering: A 334 (2002) 173-178. 
[26] V. Verma, V.K. Jindal, K. Dharamvir, Elastic moduli of a boron nitride nanotube, Nanotechnology 18 (2007) 435711.

[27] J.W. Yan, K.M. Liew, Predicting elastic properties of single-walled boron nitride nanotubes and nanocones using an atomistic-continuum approach, Composite Structures 125 (2015) 489-498.

[28] L. Jiang, W. Guo, A molecular mechanics study on size-dependent elastic properties of single-walled boron nitride nanotubes, Journal of the Mechanics and Physics of Solids 59 (2011) 1204-1213.

[29] J.R. Lukes, H. Zhong, Thermal Conductivity of Individual Single-Wall Carbon Nanotubes, Journal of Heat Transfer 129 (2007) 705-716.

[30] J.W. Jiang, J.S. Wang, Theoretical study of thermal conductivity in single-walled boron nitride nanotubes, Physical Review B 84 (2011) 085439.

[31] C. Sevik, A. Kinaci, J.B. Haskins, T. Çağın, Characterization of thermal transport in low-dimensional boron nitride nanostructures, Physical Review B 84 (2011) 085409.

[32] L. Lindsay, D.A. Broido, Optimized Tersoff and Brenner empirical potential parameters for lattice dynamics and phonon thermal transport in carbon nanotubes and graphene, Physical Review B 81 (2010) 205441.

[33] L.F.C. Pereira, B. Mortazavi, M. Makaremi, T. Rabczuk, Anisotropic thermal conductivity and mechanical properties of phagraphene: a molecular dynamics study, RSC Advances 6 (2016) $57773-57779$.

[34] B. Mortazavi, Z.Y. Fan, L.F.C. Pereira, A. Harju, T. Rabczuk, Amorphized graphene: A stiff material with low thermal conductivity, Carbon 103 (2016) 318-326.

[35] C. Sevik, A. Kinaci, J.B. Haskins, T. Çağın, Influence of disorder on thermal transport properties of boron nitride nanostructures, Physical Review B 86 (2012) 075403.

[36] A. Kinaci, J.B. Haskins, C. Sevik, T. Çağın, Thermal conductivity of BN-C nanostructures, Physical Review B 86 (2012) 115410.

[37] B. Mortazavi, Y. Rémond, Investigation of tensile response and thermal conductivity of boron-nitride nanosheets using molecular dynamics simulations, Physica E: Low-dimensional Systems and Nanostructures 44 (2012) 1846-1852.

[38] B. Mortazavi, O. Rahaman, T. Rabczuk, L.F.C. Pereira, Thermal conductivity and mechanical properties of nitrogenated holey graphene, Carbon 106 (2016) 1-8. 
[39] T. Schneider, E. Stoll, Molecular-dynamics study of a three-dimensional one-component model for distortive phase transitions, Physical Review B 17 (1978) 1302-1322.

[40] B. Mortazavi, T. Rabczuk, Multiscale modeling of heat conduction in graphene laminates, Carbon 85 (2015) 1-7.

[41] S. Ghosh, I. Calizo, D. Teweldebrhan, E.P. Pokatilov, D.L. Nika, A.A. Balandin, W. Bao, F. Miao, C.N. Lau, Extremely high thermal conductivity of graphene: Prospects for thermal management applications in nanoelectronic circuits, Applied Physics Letters 92 (2008) 151911.

[42] P.G. Klemens, Theory of Thermal Conduction in Thin Ceramic Films, Int. J. Thermophys. 22 (2001) 265-275.

[43] A. Cepellotti, G. Fugallo, L. Paulatto, M. Lazzeri, F. Mauri, N. Marzari, Phonon hydrodynamics in two-dimensional materials, Nature Communications, 6 (2015) 6400.

[44] Y. Xiao, X.H. Yan, J. Xiang, Y.L. Mao, Y. Zhang, J.X. Cao, J.W. Ding, Specific heat of single-walled boron nitride nanotubes, Applied Physics Letters 84 (2004) 4626.

[45] B. Mortazavi, L.F.C. Pereira, J.W. Jiang, T. Rabczuk, Modelling heat conduction in polycrystalline hexagonal boron-nitride films, Scientific Reports 5 (2015) 13228. 